\title{
5 Sollen wir Nutzen maximieren? Ansätze des Utilitarismus
}

\subsection{Benthams Glücksutilitarismus \\ 5.2 Systematische Fragen und Ausblick}

\section{1 | Benthams Glücksutilitarismus}

Ein höchstes Moralprinzip: Nach Humes Ansicht sind das Angenehme und das Nützliche Gegenstand moralischer Billigung. Auf Hume beruft sich auch Jeremy Bentham, der Begründer des Utilitarismus, der das Kriterium moralischer Richtigkeit in einer bestimmten Form von Nützlichkeit erblickt. Ursprünglich war der Utilitarismus nicht nur eine moralphilosophische Theorie, sondern auch politische Reformbewegung, die gesellschaftliche Institutionen gemäß der utilitaristischen Ethik reformieren wollte. Gegenwärtig steht der Utilitarismus zwar nicht mehr für eine einheitliche politische Bewegung (Kymlicka 2002, S. 46; Pettit 1993, S. 6); Versionen des Utilitarismus spielen aber in vielen gesellschaftlichen Kontexten immer noch eine Rolle. Zur Attraktivität der utilitaristischen Ethik trägt bei, dass ihr theoretischer Kern relativ einfach ist (Copp 2006, S. 21). Sie gehört zu den Formen normativer Ethik, die ihre primäre Aufgabe darin sehen, Kriterien für die Beurteilung der moralischen Richtigkeit von Handlungen festzulegen. Der klassische Handlungsutilitarismus, wie ihn Bentham vertritt, sieht dafür ein maßgebliches Kriterium vor, anhand dessen alle Handlungen, politische Maßnahmen oder Institutionen beurteilt werden sollen. In diesem spezifischen Sinn des in unterschiedlicher Bedeutung gebrauchten Begriffs lässt sich der Utilitarismus

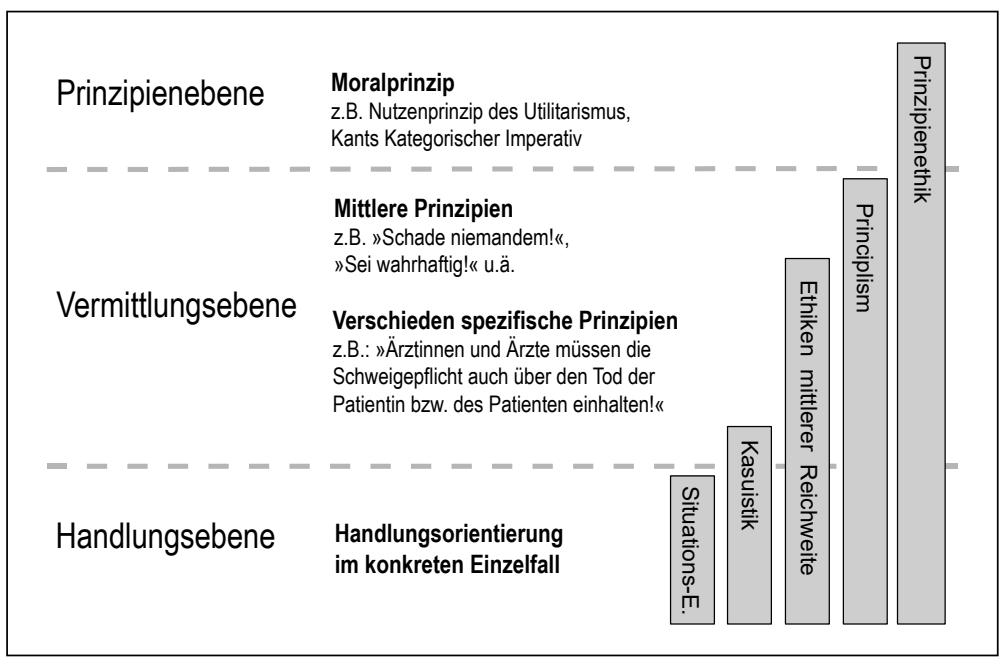

Utilitarismus als Prinzipienethik

Abb. 5.1:

Ebenen ethischer Reflexion 
Bentham 1907, "By the principle [...] of utility is meant that principle which approves or disap-

S. 2 f. proves of every action whatsoever, according to the tendency which it appears to have to augment or diminish the happiness of the party whose interest is in question: or, what is the same thing in other words, to promote or to oppose that happiness."

John Stuart Mill, Schüler Benthams und der wohl am meisten rezipierte Vertreter des Utilitarismus, formuliert den Grundgedanken des Utilitarismus ähnlich:

Mill 1963, "The creed which accepts as the foundation of morals, Utility, or the Happiness Bd. 10, S. 210 Principle, holds that actions are right in proportion as they tend to promote happiness, wrong as they tend to produce the reverse of happiness. By happiness is intended pleasure, and the absence of pain; by unhappiness, pain, and the privation of pleasure."

Gütermonismus: Grundlage des klassischen Utilitarismus, wie er von tives Wohlgefühl Bentham vertreten wird, ist eine monistische Werttheorie oder, anders gesagt, ein Gütermonismus. Gütermonist/innen nehmen an, dass es grundsätzlich nur ein einziges intrinsisches Gut gibt, also nur eines, das um seiner selbst und nicht um eines anderen Gutes willen erstrebt wird. Natürlich leugnen auch Gütermonist/innen nicht, dass es in unserem Leben eine Vielfalt unterschiedlicher erstrebenswerter Dinge gibt. Aus gütermonistischer Sicht ist diese Vielfalt jedoch ein bloßes Oberflächenphänomen. Alle erstrebenswerten Dinge, so unterschiedlich sie sein mögen, sind entweder deshalb erstrebenswert, weil in ihnen etwas von dem intrinsischen Gut enthalten ist oder sie selbst einen Teil dieses intrinsischen Gutes ausmachen, oder deshalb, weil sie Mittel zur Realisierung dieses Gutes sind. Güterpluralist/innen gehen hingegen davon aus, dass es wenigstens zwei qualitativ verschiedene, nicht aufeinander reduzierbare Güter gibt.

Hedonismus: Das vom Utilitarismus postulierte intrinsische Gut als Nutzen bezeichnet. Glücksutilitarist/innen wie Bentham setzen Nutzen mit Glück gleich. Ihr Glücksbegriff ist dabei denkbar weit entfernt von perfektionistischen oder gar moralistischen Interpretationen menschlichen Glücks, wie sie von Aristoteles oder den stoischen Philosophen vertreten werden. Benthams Glücksutilitarismus geht von einem hedonistischen Glücksbegriff aus. Er interpretiert Glück als pleasure, als einen Zustand subjektiven Wohlbefindens, bei dem sich die Waage zwischen 
Lust- und Unlustgefühlen so weit wie möglich zur Seite der Lust senkt. Damit ist Bentham zwar nicht festgelegt auf die von Aristoteles im ersten Buch der Nikomachischen Ethik verworfene Glücksauffassung, wonach Glück in sinnlichem Genuss besteht. Der klassische Glücksutilitarismus ist vielmehr völlig indifferent gegenüber der Frage, auf welche Weise subjektives Wohlempfinden erreicht wird. Ob sinnliche oder geistige Genüsse vorzuziehen sind, hängt allein von der Menge des produzierten Wohlseins ab. Was zählt, ist allerdings in jedem Fall ein subjektives Wohlgefühl. Auf qualitative Unterscheidungen verschiedener Arten dieses Wohlgefühls (die eine Aufgabe des Wertmonismus bedeuten würden) verzichtet Bentham. Entscheidend ist die Quantität (Intensität und Dauer), nicht die Qualität des subjektiv empfundenen Glücks:

"Prejudice apart, the game of push-pin is of equal value with the arts and sciences of music and poetry. If the game of push-pin furnish more pleasure, it is more valuable than either."

Universalismus: Eine auch in Hinsicht auf die historische Bedeutung des Utilitarismus wesentliche Eigenschaft dieser ethischen Position liegt in seiner Ablehnung traditioneller Privilegien und sozialer Diskriminierungen. Jedes Lebewesen, dessen Glücksempfinden einen Beitrag zum Gesamtnutzen leisten kann, verdient gleiche Berücksichtigung: Ihr oder sein möglicher Nutzenbeitrag muss genauso in die Nutzensumme einbezogen werden wie der mögliche Beitrag aller anderen Lebewesen. Da auch Tiere empfindungsfähig sind, sind sie bei der Beurteilung alternativer Handlungsoptionen ausdrücklich mit zu berücksichtigen: "the question is not, can they reason? nor, Can they talk? but, Can they suffer?« (Bentham 1907, S. 311). Bentham ist damit einer der Pioniere der Tierethik, deren Anliegen er mit dem der Befreiung von rassistischer Diskriminierung vergleicht:

"The day may come, when the rest of the animal creation may acquire those rights which never could have been withholden from them but by the hand of tyranny. The French have already discovered that the blackness of the skin is no reason why human being should be abandoned without redress to the caprice of a tormentor [...]. It may come one day to be recognized, that the number of legs, the villosity of the skin, or the termination of the os sacrum, are reasons equally insufficient for abandoning a sensitive being to the same fate."

Interindividuelle Nutzenaggregation und Gesamtnutzen: Aus Sicht des benthamschen Utilitarismus ist neben der Qualität des Glücksempfindens auch dessen Verteilung über verschiedene Individuen an sich irrelevant. Maßgeblich ist allein die Gesamtsumme des von allen empfindungsfähigen Wesen erlebten Glücks. Der Utilitarismus muss also annehmen, dass Nutzen (im Fall des Glücksutilitarismus: Glück) prinzipiell quantifizierbar, messbar und auch interindividuell vergleichbar ist, denn nur auf dieser Grundlage können verschiedene Weltzustände im Hinblick auf den in ihnen realisierten Gesamtnutzen miteinander verglichen werden. Der von Bentham vertretene Utilitarismus ist dabei als Gesamtnutzenutilita-

Intensität und

Dauer des

Glückserlebens

Bentham 1830, S. 206

Gleiche Berücksichtigung aller empfindungsfähigen Wesen

Bentham 1907, S. 311

Verteilungsindifferenz 
Instrumentelle Deutung der Moral rismus zu charakterisieren. Diese Form des Utilitarismus definiert das zu maximierende Gut unmittelbar als Summe des Glücks aller empfindungsfähigen Wesen. Daraus folgt, dass das zu maximierende Gut nicht nur durch die Erhöhung des Wohlbefindens von bereits existierenden Individuen vermehrt werden kann, sondern auch durch die Vergrößerung der Anzahl empfindungsfähiger Wesen, sofern die neu hinzukommenden Wesen mehr Lust als Unlust empfinden.

Handlungsutilitarismus und Konsequentialismus: Der von Bentham verteidigte Utilitarismus bemisst die Richtigkeit jeder konkreten Handlung an ihrem Beitrag zur Nutzenmaximierung (Bentham 1907, Kap.4). Dieser Utilitarismus ist daher als Handlungs- bzw. Akt-Utilitarismus zu bezeichnen. Akt-utilitaristische Ethiken sind zugleich konsequentialistisch.

Konsequentialistisch ist eine normativ-ethische Theorie genau dann, wenn sie die Richtigkeit jeder Handlung vollständig von deren Folgen abhängig macht. Mit dem Begriff ,Folgen` sind dabei nicht nur Ereignisse gemeint, die der seigentlichen Handlung، zeitlich nachgeordnet sind. Zu den Folgen zählen vielmehr auch die Handlungsergebnisse, die bereits Teil der Handlungsbeschreibung sind (etwa schon das Gedemütigtsein einer Person als Ergebnis einer Handlung, die als Demütigung beschrieben wird) und unmittelbar im Vollzug der Handlung auftretende Effekte. So wäre auch die im Vollzug des Tanzes erlebte Freude des Tänzers als 'Folge‘ zu bezeichnen. Die vollständige Abhängigkeit der moralischen Handlungsbewertung von den Folgen bedeutet, dass Handlungen nicht san sich bzw. sintrinsisch moralisch richtig oder falsch sein können (etwa weil sie einer gültigen Regel folgen oder widersprechen oder weil sie Ausdruck einer bestimmten Einstellung, Haltung oder Motivation sind).

Die moralische Qualität von Handlungen ist rein instrumentell zu verstehen, sie besteht in ihrem Beitrag zur Nutzenmaximierung. Da der durch eine konkrete Handlung produzierte Nutzen von den Umständen der jeweiligen Handlungssituation abhängig ist, erfordert der Handlungsutilitarismus eine situationsspezifische Folgenbewertung, die in verschiedenen Situationen unterschiedliche Handlungsvorschriften zum Ergebnis haben kann.

Maximierung: Im Sinne des utilitaristischen Maximierungsgedankens ist eine Handlung genau dann richtig, wenn zu erwarten ist, dass sie mindestens so viel Nutzen produziert wie jede andere in der betreffenden Situation mögliche Handlung. Gütermonismus und Maximierungsgedanke gemeinsam sorgen dafür, dass sich prinzipiell jedes moralische Entscheidungsproblem in eine Optimierungsaufgabe übersetzen lässt. Das ermöglicht es, alle moralischen Fragen mit derselben Methode zu lösen. Zudem sind im Rahmen einer utilitaristischen Interpretation von Handlungssituationen kaum Dilemmasituationen zu erwarten. Dilemmasituationen entstehen im Utilitarismus nur in dem unwahrscheinlichen 
Fall, dass neben der optimalen (nutzenmaximierenden) Handlungsoption noch eine zweite Option genau denselben Nutzenzuwachs verspricht. In diesem Fall können beide Optionen gleichermaßen als moralisch richtig gelten.

\section{Maximierung oder Satisfizierung?}

Nicht alle konsequentialistischen, wert- oder güterbasierten Ethiken halten die Maximierung der betreffenden Werte oder Güter für notwendig. In Konkurrenz zu Maximierungstheorien wie dem Utilitarismus stehen sogenannte Satisfizierungstheorien, die Ziele unterhalb der Nutzenmaximierung als moralisch hinreichend auszuweisen suchen, beispielsweise um die Überforderung moralischer Akteur/innen zu vermeiden (Slote/Pettit 1984).

Die von Bentham ursprünglich verwandte Formulierung vom ‘größten Glück der größten Zahl gibt den zu realisierenden Zustand übrigens nicht eindeutig an. Sie lässt sich so verstehen, als müsste neben dem Glück auch noch die Zahl der glücklichen Wesen maximiert werden. Das würde jedoch den Gütermonismus und damit die prinzipielle Entscheidbarkeit aller moralischen Probleme gefährden. Denn das Ziel, die Zahl der Glück empfindenden Wesen zu maximieren, fällt nicht notwendigerweise mit dem Ziel zusammen, die Summe des Glücks aller Wesen zu maximieren. Bentham ist später denn auch dazu übergegangen, nur noch vom 'größten Glückı zu sprechen (Scarre 1996, S. 24).

Teleologie: Der Handlungsutilitarismus ist zudem (im Sinne der Standarddefinition von Frankena und Rawls siehe Kap. 2.2) als teleologische Ethik und damit als nicht-deontologisch zu charakterisieren. Denn es handelt sich um eine Ethik, die dasjenige Handeln als moralisch richtig ausweist, das ein >vor-moralisches` Gut maximiert. Der Nutzen des benthamschen Glücksutilitarismus ist als vor-moralisches Gut zu bezeichnen, weil seine Charakterisierung als Gut nicht von moralischen, sondern von rein hedonistischen Kriterien abhängt (Bentham selbst gebraucht den Begriff `Deontologie` in einer heute nicht mehr üblichen Weise und versteht ihn wörtlich und ganz allgemein als 'Sollenslehre', so dass er ihn auch auf seinen eigenen Ansatz anwenden kann). Nicht alle konsequentialistischen Theorien sind im Sinne der von Frankena und Rawls vorgeschlagenen Definition teleologisch. Eine konsequentialistische Theorie könnte auch auf die Realisierung eines moralischen Gutes zielen, etwa auf die Herstellung sozialer Gerechtigkeit oder auf die möglichst weitreichende Entfaltung moralischer Tugenden aller Bürger/innen (vgl. Nida-Rümelin 1993).

\section{Glückskalkül und Wahrscheinlichkeit}

Nehmen wir an, eine Patientin, die nach erfolgter Operation im Krankenhaus liegt, fragt eine Pflegekraft nach dem Operationsbefund, den der zuständige Stationsarzt ihr aus Gründen, die weder der Patientin noch der Pflegekraft einleuchten, vorenthalten hat. Wie müsste sich die Pfle- 
gekraft verhalten, um im Sinne des Glücks- und Handlungsutilitarismus richtig zu handeln? Im Sinne des Konsequentialismus wären zunächst die jeweiligen Auswirkungen der verschiedenen Handlungenoptionen auf das Glück aller Betroffenen zu ermitteln. Der Glückszustand kann dabei als ein quantitativer Wert, beispielsweise als Punkt auf einer linearen Lust-Unlust-Skala gedacht werden. Die Ergebnisse könnten dann im Rahmen eines Glückskalküls verrechnet werden, in dem für jede Handlungsoption jeweils die mit ihr verbundenen Werte zusammengezählt werden. Moralisch richtig ist diejenige Handlungsweise, welche die größte Glückssumme zur Folge hat.

\begin{tabular}{|l|l|l|l|l|}
\hline & Patientin & Pflegekraft & Vorgesetzter & Summe \\
\hline Wahrhaftigkeit & 30 & 45 & 85 & 160 \\
\hline $\begin{array}{l}\text { Unwahrhaftig- } \\
\text { keit }\end{array}$ & 40 & 55 & 60 & 155 \\
\hline
\end{tabular}

Nehmen wir an, eine wahrhaftige Aussage über den Operationsbefund würde die Patientin psychisch belasten und ihr Befinden vergleichsweise negativ beeinflussen. Die Pflegekraft wäre durch ihre Wahrhaftigkeit zwar von Gewissensskrupeln entlastet, hätte aber mit Sanktionen ihres Vorgesetzten zu rechnen, was zu Minderung ihres Glücks führt. Der Vorgesetzte schließlich hegt der Pflegekraft gegenüber ohnehin negative Gefühle und ist froh über die Gelegenheit, sie zu maßregeln, was sein Wohlbefinden deutlich steigert. Hat man die Auswirkungen der verschiedenen Handlungsoptionen auf das Glück aller Betroffenen erst einmal so weit ermittelt, muss man lediglich die verschiedenen Glückserträge jeder Handlungsoption summieren und diejenige wählen, die die höchste Gesamtsumme erbringt.

Ausgeklammert wurde bislang die Wahrscheinlichkeitsverteilung. Grundsätzlich lassen sich Handlungsfolgen immer nur mit einer gewissen Wahrscheinlichkeit prognostizieren. In Fällen, in denen Wahrscheinlichkeitswerte angesetzt werden können, besteht eine mögliche Strategie darin, die Wahrscheinlichkeit, mit der ein bestimmtes Handlungsszenario einen bestimmten Nutzenertrag realisiert, jeweils mit diesem Nutzenertrag zu multiplizieren. Ein Glückszuwachs von 5, der mit $50 \%$ iger Wahrscheinlichkeit erzielt werden kann, wäre z. B. im Glückskalkül mit dem Wert 2,5 zu berücksichtigen. Obige Tabelle wäre dann so zu lesen, dass die verschiedenen Zahlenwerte nicht einfach Nutzenwerte, sondern Nutzenerwartungswerte darstellen, die jeweils aus der Summe der Produkte verschiedener möglicher Nutzenerträge mit der Wahrscheinlichkeit ihrer jeweiligen Realisierung gebildet sind. Diese Vorgehensweise stößt an Grenzen, wenn die Eintrittswahrscheinlichkeiten der relevanten Ereignisse nicht mehr hinreichend verlässlich zu ermitteln sind, wenn wir es also nicht mit berechenbaren Chancen und Risiken, sondern mit Ungewissheiten zu tun haben (klassisch Knight 1921). 


\section{2 | Systematische Fragen und Ausblick}

\subsection{1 | Warum Utilitarismus?}

Klarheit und Sparsamkeit: Für den Utilitarismus spricht seine Klarheit und Einfachheit. Anhand eines einzigen Prinzips sollen alle moralischen Problemsituationen interpretiert und alle verfügbaren Handlungsoptionen im Vergleich zueinander bewertet werden. Die Sparsamkeit der Theorie beschränkt sich jedoch nicht auf ihre innere Struktur, sondern betrifft auch die von ihr in Anspruch genommenen Voraussetzungen. Die Theorie als solche kommt ohne anspruchsvolle Annahmen über die Natur der praktischen Rationalität aus. Ein weiterer theoretischer Vorzug besteht darin, dass der Utilitarismus mit unterschiedlichen metaethischen Positionen vereinbar, also auch in dieser Hinsicht nicht allzu voraussetzungsvoll ist. Unter den Vertretern des Utilitarismus befinden sich Anhänger des intuitionistischen und des naturalistischen Realismus ebenso wie solche des präskriptivistischen Nonkognitivismus. Wie angedeutet versprechen dabei die Beschränkung auf ein Moralprinzip, der Gütermonismus und der Konsequentialismus, alle moralischen Konflikte in Optimierungsfragen $\mathrm{zu}$ transformieren, für die es eindeutig entscheidbare Antworten gibt. Bei der Beantwortung dieser Fragen kann sich die utilitaristische Ethik auf wissenschaftliche Verfahren der Folgenabschätzung, Ökonomie, Entscheidungs- und Spieltheorie stützen.

Utilitarismus und Unparteilichkeit: Einfachheit, Klarheit und theoretische Sparsamkeit reichen freilich nicht aus, um normativ-ethische Prinzipien oder Theorien attraktiv erscheinen zu lassen. Vielmehr wird in aller Regel erwartet, dass sie auch vor dem Hintergrund wohlerwogener und weithin geteilter moralischer Intuitionen plausibel sind (siehe Kap. 7.2.2, Kap. 8.2 und Kap. 9.5) und/oder dass eine noch tiefergehende Begründung für die von den betreffenden Prinzipien oder Theorien erhobenen Ansprüche gegeben wird. Vertreter/innen des Utilitarismus setzen dabei in aller Regel an zwei Punkten an: Erstens argumentieren sie, dass rationales Handeln grundsätzlich nutzenorientiert ist. Zweitens stützen sie sich auf die moralische Intuition der Unparteilichkeit. Nehme man beides - das Nutzenstreben als allgemeines Kennzeichen rationaler Handlungsorientierung und die Unparteilichkeit - zusammen, so gelange man mehr oder weniger zwangsläufig zur utilitaristischen Forderung, den Gesamtnutzen (in dem ja die individuellen Nutzenbeiträge aller empfindungsfähigen Wesen unparteilich berücksichtigt sind) zu maximieren (z. B. Smart 1973).

Mills 'Beweis، des Utilitarismus: In einer viel diskutierten Passage stützt sich auch John Stuart Mill auf diese beiden Elemente, um einen "Beweis« (proof) des Utilitarismus zu entwickeln. Er stellt zunächst fest, dass Aussagen über `letzte Ziele - Dinge, die als solche erstrebenswert und also gut sind - nicht beweisbar seien; ebenso wenig wie Axiome (first principles) auf anderen Wissensgebieten (Mill 1963, Bd. 10, S. 234). Ebenso wie die Sichtbarkeit oder Hörbarkeit von etwas einzig dadurch zu belegen ist, dass es tatsächlich gesehen oder gehört wird, kann der Charakter des Erstrebenswerten nur dadurch belegt werden, dass es tatsäch-

Nutzenstreben und Unparteilichkeit 
Mill 1963, Bd. 10, S. 234

Angesehen und erstrebenswert

Mill 1963, Bd. 10, S. 237 f. einziges Gut lich erstrebt wird. Nun zeigt sich, dass alle Menschen ihr eigenes Glück anstreben. Gemäß der Analogie der optischen oder akustischen Wahrnehmung dürfen wir daraus folgern, dass das Glück tatsächlich erstrebenswert ist. Weil nun für jedes Individuum gelte, dass sein eigenes Glück für dieses Individuum gut sei, sei das allgemeine Glück für die Gesamtheit aller Personen ein Gut:

"No reason can be given why the general happiness is desirable, except that each person, so far as he believes it to be attainable, desires his own happiness. This, however, being a fact, we have not only all the proof which the case admits of, but all which it is possible to require, that happiness is a good: that each person's happiness is a good to that person, and the general happiness, therefore, a good to the aggregate of all persons."

Glück als Damit sei allerdings erst gezeigt, dass Glück eines der Ziele menschlichen Verhaltens und damit eines der Moralkriterien sei (ebd.). In einem zweiten Schritt möchte Mill daher nachweisen, dass Glück das einzige intrinsische Gut darstellt. Während die Frage, ob Glück erstrebenswert ist, wenig strittig scheint, ist die These, Glück sei das einzige intrinsische Gut, weitaus strittiger. Mill versucht allerdings nicht, nachzuweisen, dass alle anderen Güter rein instrumenteller Natur sind. Vielmehr vertritt er eine inklusive Deutung des utilitaristischen Glücksbegriffs, der zufolge verschiedene Dinge, die um ihrer selbst willen erstrebt werden, als konstitutive Elemente des Glücks verstanden werden (vgl. die inklusive Deutung des aristotelischen Lebensziels in Kap.2.3.2). So könnten Musik, Gesundheit oder auch Tugend nicht nur instrumentell, sondern auch um ihrer selbst willen erstrebt werden. Mills Auffassung ist, dass alle entsprechend intrinsisch wertvollen Güter sich $\mathrm{zu}$ dem einen übergreifenden Gut >Glück zusammenschließen. Aber warum sollten wir das annehmen? Was stellt die grundsätzliche Homogenität der verschiedenen Güter sicher, und warum ist gerade Glück der passende Begriff dafür? Mill hält es für eine analytische, allein aus der Bedeutung der betreffenden Begriffe folgende, Wahrheit, dass wir uns, was wir als erstrebenswert (desirable) denken, auch als angenehm (pleasant) vorstellen. Er führt aus,

"that desiring a thing and finding it pleasant, aversion to it and thinking of it as painful, are phenomena entirely inseparable, or rather two parts of the same phenomenon; in strictness of language, two different modes of naming the same psychological fact: that to think of an object as desirable (unless for the sake of its consequences), and to think of it as pleasant, are one and the same thing; and that to desire anything, except in proportion as the idea of it is pleasant, is a physical and metaphysical impossibility."

Probleme des millschen Beweises: Mills >Beweis` der Gültigkeit des Utilitarismus ist überwiegend kritisch aufgenommen worden. Einwände beziehen sich dabei auf die folgenden Schritte:

1. den Übergang vom Erstrebtwerden zum Erstrebenswert-Sein;

2. den Übergang vom individuellen Glücksstreben zum Erstreben des allgemeinen Glücks; 
3. die Annahme, dass Glück nicht nur ein Gut, sondern das einzige Gut darstellt.

Erstrebtwerden und Erstrebenswert-Sein: Gegen den Schluss vom Erstrebtwerden auf das Erstrebenswert-Sein ist eingewandt worden, dass faktisch weder alle erstrebenswerten Dinge erstrebt würden noch alle erstrebten Dinge tatsächlich erstrebenswert seien. Gegen Mills Vergleich des Verhältnisses zwischen dem Erstrebtwerden und dem Erstrebenswert-Sein mit dem Verhältnis zwischen dem Gesehen- bzw. Gehörtwerden und dem Sichtbar- bzw. Hörbarsein wird unter anderem vorgebracht, dass er aufgrund einer Äquivokation des Begriffs »desirable« unhaltbar sei. »Desirability« könne nämlich sowohl als die Eigenschaft des Erstrebtwerden-Könnens als auch als die des Erstrebenswert-Seins verstanden werden. Mills Analogie mit der Sicht- oder Hörbarkeit sei nur in der ersten Bedeutung haltbar; für sein Argument benötige er aber die zweite Bedeutung des Erstrebenswert-Seins (Moore 1959, S.66f.). Allerdings vertritt Mill nicht die Auffassung, dass Erstrebtwerden und Erstrebenswert-Sein identisch sind. Er vertritt nur die schwächere These, dass das Erstrebtwerden die einzige Evidenz für das Erstrebenswert-Sein darstellt. Und während es sehr gute Gründe gibt, zu bestreiten, dass alles, was im Einzelfall erstrebt wird, tatsächlich erstrebenswert ist, gibt es weniger gute Gründe zu bestreiten, dass etwas, das (wie nicht nur Mill annimmt) von allen Personen erstrebt wird, tatsächlich erstrebenswert ist - also zu behaupten, dass die gesamte Menschheit in dieser Hinsicht verblendet oder irrational ist. Der erste Schritt des millschen Arguments kann also zwar nicht die Annahme beweisen, dass Glück erstrebenswert ist, er liefert aber durchaus gute Gründe dafür.

Individuelles und allgemeines Glück: Gegen den zweiten Schritt ist vielfach eingewandt worden, dass er einen Fehlschluss von den Teilen auf das Ganze (fallacy of composition) beinhalte. Auch der Utilitarist Sidgwick hält Mills Überlegung für unhaltbar:

"For an aggregate of actual desires, each directed towards a different part of the general happiness, does not constitute an actual desire for the general happiness, existing in any individual; and Mill would certainly not contend that a desire which does not exist in any individual can possibly exist in an aggregate of individuals."

Nicht alles Erstrebte ist erstrebenswert

Sidgwick 1907, S. 388

"Fallacy of composition" folgt nicht, dass das allgemeine Glück ein Gut für die Gemeinschaft aller Personen wäre. Der Umstand, dass in einer von Anna und Rosa bewohnten Welt Anna nach dem Glück Annas strebt und Rosa nach dem Glück Rosas strebt, impliziert weder, dass Anna und Rosa eine Gemeinschaft bilden, die nach dem Gesamtglück Annas und Rosas streben müsste, noch dasjenige, was das utilitaristische Nutzenprinzip tatsächlich fordert, nämlich dass sowohl Anna als auch Rosa als Individuen jeweils nach dem Gesamtglück Annas und Rosas streben müssten. Allerdings zielt Mills Argument nicht darauf, nachzuweisen, dass alle Personen faktisch das allgemeine Glück anstreben. Sein Argument soll vielmehr zeigen, dass wir - da wir (die wir alle das eigene Glück anstreben) der Überzeugung 
sind, dass Glück ein Gut darstellt - Grund haben, dieses Gut auch dort zu realisieren, wo es nicht unser eigenes ist. Auch dieses Ziel könnte Mills Argument jedoch nur erreichen, wenn es den Nachweis erbringen könnte, dass Glück einen rakteursneutralen` Wert darstellt, also etwas, das nicht nur aus einer bestimmten partikularen Perspektive als gut erscheint, sondern aus schlechthin jeder Perspektive (vgl. Nagels analogen Versuch, nachzuweisen, dass Schmerz ein akteursneutrales Übel ist; Nagel 1986, Kap. 8). Dies ist jedoch zumindest nicht ohne Weiteres ersichtlich (für eine Verteidigung des millschen Arguments vgl. Sayre-McCord 2001).

Glück als einziges Gut: Fragen wirft auch Mills These auf, dass wir alles aus unserer Sicht Erstrebenswerte auch als angenehm denken. Könnte es eine Pflegekraft nicht für erstrebenswert halten, ihren Patienten wahrhaftig über dessen Gesundheitszustand zu informieren, obwohl sie davon kurz- wie langfristig sowohl für sich selbst als auch für den Patienten eher eine Verminderung des Wohlbefindens erwartet? Und könnte sie nicht vielleicht sogar dann an dieser Einstellung festhalten, wenn sie wüsste, dass ihr nach Erteilung einer unwahrhaftigen Auskunft eine Droge verabreicht würde, die jeden Anflug von Gewissensqualen erfolgreich unterdrücken würde (vgl. dazu die Überlegungen zu Grenzen des Hedonismus in Kap. 2.3.2)? Falls man diese Fragen bejaht, kann man als Utilitarist/in zwar weiter zu zeigen versuchen, dass die Haltung der Pflegekraft irrational ist. Man kann sich dabei aber nicht mehr auf die von Mill vertretene These stützen, es stelle eine allgemein akzeptierte analytische Wahrheit dar, dass »erstrebenswert sein« und »Wohlbefinden erzeugend " gleichbedeutend sind.

Hedonistische Motivationstheorie

Angenehmes und Erstrebenswertes: Die Bedeutungsidentität der Begriffe der »desirablility" und "pleasurability« zu behaupten, ist nicht die einzige Möglichkeit, zu begründen, dass das einzig erstrebenswerte Gut in demjenigen liegt, das Wohlbefinden erzeugt. Dieselbe Folgerung ließe sich etwa auch aus einer hedonistischen Motivationstheorie ziehen, die annimmt, dass die einzige Ursache dafür, bestimmte Dinge anzustreben in der Erwartung liegt, dadurch unsere Lust/Unlust-Bilanz zu verbessern. Diese Theorie sieht sich allerdings starken Begründungslasten ausgesetzt. Auch würde sie auf eine rein instrumentelle Interpretation aller Güter außer dem psychologischen Wohlgefühl selbst hinauslaufen. Sie widerspräche wohl auch der von Mill vertretenen inklusiven Lesart des Glücks, das die Möglichkeit einschließt, dass wir bestimmte Dinge (etwa Tugend) um ihrer selbst willen wertschätzen können. Eine andere Verteidigungsmöglichkeit könnte in dem Versuch liegen, den Begriff "pleasurable« so zu interpretieren, dass seine Bindung an ein deutlich feststellbares psychologisches Gefühl gelockert wird. Ein Vorbild für diese Strategie liegt in Humes Behauptung, dass unsere Handlungen von »calm passions « mitbestimmt werden, die wir gar nicht als solche wahrnehmen, sondern nur aus ihren Wirkungen erschließen können (siehe Kap. 4.1). Diese Antwort birgt indes die Gefahr, dass die Theorie unüberprüfbar wird: Wenn wir nicht mehr unabhängig von den zu erklärenden Entscheidungen und Präferenzen ermitteln können, was Akteur/innen als angenehm empfinden, läuft die Erklärung der Strebungen von Personen durch deren Wahrnehmung des Erstrebten als angenehm auf eine Scheinerklärung nach dem Muster der 
molièreschen virtus dormitiva hinaus. Für die utilitaristische Ethik brächte die Antwort zudem das Problem mit sich, dass das Gefühl des Angenehmen dann nicht mehr als ein identifizierbares, messbares und homogenes Gut gedacht werden kann. Eine naheliegende Konsequenz wäre dann der Übergang vom Glücks- zum Präferenzutilitarismus (s. u.).

Reduktion der Begründungsansprüche: Angesichts der genannten Schwierigkeiten versuchen die meisten Vertreter/innen des Utilitarismus nicht, diese Theorie als gültig zu beweisen, sondern begnügen sich mit dem Versuch, ihre Plausibilität zu verdeutlichen. Auch wenn Mills Schritt vom individuellen Glückstreben zum Wert des Gesamtglücks nicht ohne Weiteres überzeugend ist, scheint er zumindest geeignet, Intuitionen der Unparteilichkeit zu mobilisieren, die weit verbreitet und nach Ansicht vieler Interpret/innen für jedes plausible Moralverständnis grundlegend sind.

\subsection{2 | Widerstreitende Intuitionen}

Moralische Intuitionen: Der Versuch, den Utilitarismus auf vorphilosophische Intuitionen zu stützen, ist allerdings nicht unproblematisch. Intuitionen der Unparteilichkeit etwa sind zwar weit verbreitet, lassen sich aber (wie auch in den folgenden Kapiteln deutlich werden wird) sehr unterschiedlich interpretieren. Hinsichtlich des Gütermonismus, Konsequentialismus und Hedonismus sprechen unsere vortheoretischen Intuitionen ebenfalls nicht unbedingt für den klassischen Utilitarismus. Ein unklares Bild ergibt sich jedenfalls im Hinblick auf mögliche Implikationen und Konsequenzen der utilitaristischen Ethik in verschiedenen Fallbeispielen. Dass eine Konzeption normativer Ethik gegen bestimmte moralische Intuitionen verstößt, ist nicht unbedingt ein Argument gegen diese Theorie, zumal vortheoretische Intuitionen teils intern widersprüchlich sind und sich im Lauf der historischen Entwicklung (teils unter dem Einfluss ethischer Theorien) auch gewandelt haben. Wohl aber schwächt es die Plausibilität ethischer Theorien zumindest in dem Maß, in dem diese selbst mit dem Hinweis auf bestimmte moralische Intuitionen begründet werden sollen (vgl. die Ausführungen zum Überlegungsgleichgewicht, zum Intuitionismus und zur Berufung auf faktische Konsense in Kap. 7, Kap. 8 und Kap. 9).

Verantwortlichkeit und Integrität: Einige Kritiker/innen des Utilitarismus befürchten eine Auflösung persönlicher Verantwortlichkeit und Integrität durch den Utilitarismus. Ein erster Grund dafür liegt im konsequentialistischen Charakter der Theorie. Im Rahmen einer reinen Folgenorientierung ist es gleichgültig, ob ich bestimmte Konsequenzen durch eigenes Handeln direkt hervorbringe, oder dadurch, dass ich andere zu bestimmten Handlungen veranlasse. Bernard Williams führt in diesem Zusammenhang das Beispiel eines Chemikers an, der die Entwicklung chemischer Waffen leidenschaftlich ablehnt, nun aber vor der Frage steht, ob er im Rahmen eines auch aus familiären Gründen dringend benötigten Jobs an deren Entwicklung mitwirken soll - zumal angesichts der Tatsache, dass ein anderer Chemiker diese Arbeit möglicher-

Konsequenzen des Konsequentialismus 
Kein Freiraum des bloß Erlaubten

Keine ran sich، (un-)moralischen Handlungen weise mit weit größerem Enthusiasmus vorantreiben würde (Williams 1973, S. 97 f.). Zweitens verweist er auf das Beispiel eines Botanikers, der als Feldforscher in Südamerika von dem Anführer einer paramilitärischen Gruppe vor die Alternative gestellt wird, entweder selbst einen der von den Paramilitärs gefangengenommenen Indios zu erschießen, worauf der Rest der Indios freigelassen würde, oder zuzulassen, dass alle Indios erschossen werden (ebd., S. 98 f.). Führt es nicht zu einer Auflösung persönlicher Verantwortlichkeit und Integrität, wenn solche Entscheidungen schlicht im Sinne der Nutzenmaximierung getroffen werden (ebd., S. 108 ff.; zur Diskussion Ashford 2000; Moseley 2014)? Benthams rein quantitativer Glücksutilitarismus oder Richard M. Hares Präferenzutilitarismus verschärfen das Integritätsproblem insofern, als sie einen Akteur nötigen, im Rahmen der Nutzenkalkulation alle Formen subjektiven Wohlbefindens gleichermaßen zu berücksichtigen, einschließlich etwa der Schadenfreude des fanatischen Rassisten oder der Erleichterung des religiösen Fundamentalisten über die Diskriminierung der Familie oder der Religion des Akteurs.

Überforderung: Im Zusammenhang mit der Frage der persönlichen Integrität wird unter der Überschrift (over-)demandingness auch diskutiert, ob der Akt-Utilitarismus nicht auch zu einer motivationalen Überforderung der handelnden Personen führt. Die Alltagsmoral und viele Konzeptionen normativer Ethik sehen neben dem Bereich des moralisch Gebotenen und Verbotenen einen Bereich des bloß Erlaubten vor. Sie erkennen moralischen Akteur/innen also Spielräume freien Entscheidens $\mathrm{zu}$, in denen sie von moralischen Pflichten entlastet sind. Häufig wird zudem angenommen, dass in diesen Bereich des bloß Erlaubten nicht nur moralisch indifferente Handlungen fallen, sondern auch sogenannte supererogatorische Handlungen, die nicht eigentlich geboten sind (weil sie mit zu großen Zumutungen an die handelnden Personen verbunden wären), aber gleichwohl als moralisch vorbildlich gelten können. Sich als unbewaffneter Passant einem mit Schusswaffen versehenen Amokläufer in den Weg zu stellen, in der Hoffnung, zwei anderen Personen zur Flucht zu verhelfen, könnte demnach als supererogatorische Handlung verstanden werden - als moralisch heldenhaft und vorbildlich, aber nicht strikt geboten. Der utilitaristische Optimierungsgedanke hat demgegenüber zur Folge, dass es, von wenigen Ausnahmen abgesehen, nur gebotene und verbotene Handlungsoptionen gibt. Lediglich in dem unwahrscheinlichen Fall, dass mit zwei oder mehr Handlungsoptionen genau dieselben maximalen Nutzenerwartungen verbunden sind, ist die Wahl einer dieser Optionen in das Belieben der oder des Handelnden gestellt. Zugleich sind alle Handlungen moralisch relevant, die irgendeinen Einfluss auf den Gesamtnutzen haben. Sogar die Entscheidung, ob ich Vanille- oder Erdbeereis bestelle, ist schon eine moralische Frage, wenn zu erwarten ist, dass ich aus einer der beiden Sorten mehr Genuss ziehe - denn auch mein eigenes Glück fließt ja in die Nutzensumme ein.

Intrinsische moralische Qualitäten von Handlungen: Die Alltagsmoral nimmt vielfach an, dass bestimmte Handlungen als solche - als Handlungen eines bestimmten Typs - richtig oder falsch sind, auch unabhängig von den in der spezifischen Situation erwartbaren Konsequenzen. 
Das muss nicht zwangsläufig bedeuten, dass diese Handlungen unter allen Umständen richtig oder falsch wären (im Sinne ausnahmslos gültiger, sabsoluter moralischer Regeln), sondern nur, dass ihr moralischer Charakter nicht vollständig von den in der konkreten einzelnen Handlungssituation zu erwartenden Konsequenzen abhängt (im Sinne normativer Prima-facie-Regeln; s. u.). Beispielsweise wird häufig angenommen, dass es ran sich ‘ falsch ist, zu lügen oder dass es san sich geboten ist, Versprechen zu halten, auch wenn die Konsequenzen dieser Handlungen im konkreten Einzelfall nicht optimal sein mögen. Diese Vorstellung verträgt sich jedoch nicht mit dem handlungsutilitaristischen Konsequentialismus (zum Kontext Nida-Rümelin 1993).

Individuelle Grundrechte: Bestandteil des modernen Moralverständnisses ist zudem, dass bestimmte Grundrechte von Individuen entweder gar nicht oder nur in Extremfällen aufgrund von Nützlichkeitsüberlegungen relativiert werden dürfen. Der Handlungsutilitarismus kennt demgegenüber im Grundsatz nur ein einziges individuelles Recht: Das Recht darauf, dass der eigene Beitrag zum Gesamtnutzen ebenso in die Kalkulation dieses Nutzens einbezogen wird wie die Nutzenbeiträge aller anderen Betroffenen. Aufgrund der interindividuellen Aufsummierung der Nutzenbeiträge aller Betroffenen sind jedoch mit diesem Recht keine spezifischeren persönlichen Garantien verbunden. Es ist daher vergleichsweise einfach, Fallbeispiele zu konstruieren, in denen der Handlungsutilitarismus zu Handlungen verpflichten würde, die im Rahmen der Alltagsmoral (und im Rahmen des geltenden Rechtssystems) klar verboten wären: Müsste der Handlungsutilitarismus nicht fordern, eine reiche, aber depressive Person zu töten, insofern dies schmerzfrei und unentdeckt möglich wäre, und das Erbe in anderen Händen erwartbarerweise mehr Glück produzieren würde? Etc.

Implikationen für die Güterverteilung: Auch wo nicht grundlegende Interessen von Beteiligten zur Disposition stehen, befindet sich die vom Utilitarismus vorgesehene interindividuelle Nutzenaggregation in Konflikt zu verbreiteten Intuitionen der Gerechtigkeit. Diese legen nahe, dass die Verteilung von Nutzen und Nachteilen zwischen verschiedenen Betroffenen moralisch von großer Bedeutung ist, während sie im Utilitarismus - jedenfalls unmittelbar - keine Rolle spielt. Allerdings spricht auch das utilitaristische Prinzip der Nutzenmaximierung unter bestimmten Umständen dafür, Ressourcen gleichmäßiger zu verteilen. Angesichts der extremen Ungleichheit der globalen Vermögensverteilung würde eine Umverteilung zugunsten des ärmeren Teils der Weltbevölkerung prima facie den Gesamtnutzen erhöhen. Denn dem Gesetz des abnehmenden Grenznutzens zufolge nimmt der individuelle Nutzen zusätzlicher Ressourcen mit zunehmendem Wohlstand der Ressourcenbezieher tendenziell ab. Wer zu den etwa 750 Millionen Menschen gehört, die in extremer Armut leben (also nach der Definition der Weltbank pro Tag weniger als das globale Kaufkraftäquivalent zur Verfügung hat, das 1,90 US-Dollar in den USA des Jahres 2011 entspricht), wird von einem täglichen Zusatzeinkommen von 10 Dollaräquivalenten unvergleichlich viel mehr profitieren als ein/e deutsche Durchschnittsverdiener/in oder gar eine jener reichsten 42 Personen, die gemeinsam etwa so viel besitzen wie die gesamte ärmere

Kein intrinsischer Wert der Verteilungsgerechtigkeit 
Hälfte der Weltbevölkerung (Pimentel et al. 2018). Die empirische Glücksforschung liefert starke Anhaltspunkte dafür, dass die Bedeutung materieller Güter für die individuelle Lebenszufriedenheit zumindest deutlich abnimmt oder sich gar völlig erschöpft, sobald grundlegende Bedürfnisse an Nahrung, Kleidung, Wohnung, Gesundheitsversorgung, Bildung und den materiellen Grundvoraussetzungen sozialer Partizipation einmal gedeckt sind. Utilitarist/innen wie Peter Singer (2009) fordern denn auch vehement eine gleichmäßigere globale Güterverteilung und halten die meisten Bewohner/innen wohlhabender Industriestaaten für moralisch verpflichtet, Geld für die globale Armutsbekämpfung zu spenden.

Verteilungsindifferenz des Utilitarismus: Der Zusammenhang zwischen dem utilitaristischen Gebot der Nutzenmaximierung und der gleichmäßigen Ressourcenverteilung ist jedoch kontingenter Natur. Ob Nutzenerwägungen eher eine gleichmäßige oder ungleiche Ressourcenverteilung nahelegen, hängt von den jeweiligen Situationsumständen ab. Auch braucht man nicht auf unrealistische Gedankenexperimente (wie die Idee eines übermenschlichen Glücksgefühle fähigen und extrem ressourcenverschlingenden »Nutzenmonsters«; Nozick 1974, S.41; dazu kritisch Parfit 2004, S. 10) Bezug zu nehmen, um Beispiele zu finden, in denen der Utilitarismus prima facie eine inegalitäre, intuitiv ungerecht erscheinende Ressourcenverteilung fordert. Nehmen wir beispielsweise an, dass es nur mit sehr großem Ressourcenaufwand möglich wäre, die Lebensumstände einer über viele Generationen diskriminierten und marginalisierten Bevölkerungsgruppe so zu verbessern, dass sich dies deutlich in der Glücksbilanz der Betroffenen oder gar im Gesamtnutzen niederschlägt (etwa weil dafür Institutionen grundlegend umgestaltet und fest verankerte kulturelle Klischees und soziale Verhaltensmuster transformiert werden müssten). In diesem Fall könnte es im Sinne der Nutzenmaximierung schlicht effizienter sein, Ressourcen für die Verbesserung des Glückszustandes anderer Personen einzusetzen. Selbst die Aufrechterhaltung unbegründeter kultureller Klischees, die die Verantwortung für das Elend der unterprivilegierten Gruppe deren Mitgliedern selbst zuschreiben, könnte unter bestimmten Bedingungen gesamtnutzenerhöhend wirken, wenn sie die bessergestellten Mitglieder der Gesellschaft von Schuldgefühlen entlastet. Unter solchen Bedingungen dem Nutzenprinzip zu folgen, erscheint jedoch intuitiv verwerflich und empörend. Nutzenerhöhende und moralisch gebotene Handlungsstrategien scheinen nicht identisch zu sein.

\subsection{3 | Varianten des Utilitarismus}

Im vorigen Abschnitt wurden Einwände skizziert und Intuitionen mobilisiert, die entweder gegen den Utilitarismus insgesamt oder gegen die klassische Form des Utilitarismus ins Feld geführt worden sind. Im Folgenden sollen Varianten des Utilitarismus betrachtet werden, die zum Teil mit dem Ziel entwickelt worden sind, jenen Einwänden und moralischen Intuitionen besser gerecht zu werden als der von Bentham vertretene klassische Utilitarismus. 


\section{Alternative Nutzendefinitionen}

Qualität oder Quantität: Mill wendet sich gegen Benthams These, dass alle Arten des Glückserlebens als gleichwertig zu betrachten seien, so dass nur quantitative, aber keine qualitativen Unterschiede im Glückserleben zu berücksichtigen wären:

"It is better to be a human being dissatisfied than a pig satisfied; better to be Socrates dissatisfied than a fool satisfied. And if the fool, or the pig, is of a different

Mill 1963,

Bd. 10, S. 212 opinion, it is because they only know their own side of the question. The other party to the comparison knows both sides."

Besser sind die Formen des Glückserlebens, die erfahrene Beurteiler/ innen vorziehen würden. Die Annahme, dass sich positive emotionale Erfahrungen nicht nur im Hinblick auf ihre Intensität unterscheiden, sondern dass es auch Unterschiede in ihrer Tiefe und Bedeutung gibt, erscheint plausibel. Sie nimmt der utilitaristischen Theorie allerdings die Einfachheit und Eindeutigkeit, die aus der Kombination von Gütermonismus und Konsequentialismus resultiert, denn Mills Vorschlag gemäß gibt es nun zwei Dimensionen (Quantität und Qualität des Gesamtglücks), im Hinblick auf die optimiert werden kann. Um die Klarheit der Orientierung wiederherzustellen, könnte zwar ein Umrechnungsfaktor eingeführt werden, der Qualität und Quantität in Beziehung setzt. Für zusätzliche Schwierigkeiten sorgt jedoch die Bezugnahme auf »erfahrene Beurteiler/ innen«, zumal Mill einräumen muss, dass diejenigen, die Erfahrungen mit verschiedenen Glücksformen gemacht haben, faktisch nicht immer die wertvolleren vorziehen, was durch eine Art Abstumpfung zu erklären sei. Wir sind daher auf Beurteilerinnen und Beurteiler angewiesen, die nicht nur verschiedene Arten des Glücks erleben, sondern ihre »nobleren Gefühle« auch gepflegt und am Leben erhalten haben (Mill 1993, Bd. 10, S. 212 f.). Dabei droht freilich ein Zirkel, wie er bereits im Kontext der aristotelischen Ethik diskutiert wurde (siehe Kap. 2.2): Wir benötigen bereits ein Kriterium, um zu beurteilen, was die "nobleren Gefühle« sind, um festzustellen, wer als Beurteiler/in in Frage kommt.

Negativer Utilitarismus: Der klassische Utilitarismus betrachtet Glück und Leid als Kontinuum, so dass das Kalkül der Glücksmaximierung die Vermehrung des Glücks und die Minderung des Unglücks nicht gesondert in Rechnung stellen muss. Vertreter/innen des sogenannten Negativen Utilitarismus halten es jedoch für plausibel, dass die Verpflichtung zur Vermeidung oder Linderung von Leiden grundsätzlich Vorrang vor der Pflicht haben sollte, Glück zu maximieren (Walker 1974). Durch die Einführung eines entsprechenden Vorrangprinzips hoffen sie, auch Intuitionen der Gerechtigkeit besser gerecht zu werden. Tatsächlich sind auch viele nicht-utilitaristische Autor/innen der Auffassung, dass die Minderung von Leiden Vorrang vor der Mehrung des Glücks haben sollte (so nachdrücklich Gert 1998).

Glück oder Präferenzen: Wie Bentham hält auch Mill an der Vorstellung fest, dass das zu maximierende Gut letztlich das Glück der Betroffenen ist. Benthams und Mills Utilitarismus ist daher als Glücksutilitaris-
Arten des Glücks

Vorrang der Leidvermeidung? 
Präferenzbefriedigung als Nutzen? mus zu charakterisieren: Was moralisch zählt, sind die erwartbaren Folgen fraglicher Handlungen für die objektive Glücksmenge (auch wenn diese sich an Intensität und Dauer subjektiv erlebter Gefühlszustände bemisst), nicht hingegen die Folgen für das Ausmaß der Befriedigung subjektiver Präferenzen. Vereinfacht gesagt: Benthamsche Moralakteur/ innen müssen tun, was objektiv dazu beiträgt, die Handlungsbetroffenen (einschließlich ihrer selbst) glücklich zu machen, nicht etwa, was diese Betroffenen am liebsten hätten. Etliche Utilitarist/innen haben später jedoch vorgeschlagen, nicht die Maximierung des hedonistisch interpretierten Gesamtglücks, sondern die Maximierung der Befriedigung von Verlangen (desires) oder Präferenzen als Ziel moralisch richtigen Handelns zu verstehen. Für den Übergang vom Glücksutilitarismus zum Präferenzutilitarismus werden sowohl methodologische als auch normative Gründe angeführt:

1. Die Intensität des erlebten Glücks Anderer - zumal einer großen Zahl - lässt sich schlecht auf kontrollierte Weise ermitteln. Für die Feststellung von Präferenzen durch direkte Befragungen oder durch Rückschlüsse aus tatsächlichem Verhalten stehen hingegen gut etablierte - wenngleich im Hinblick auf ihre jeweilige Aussagekraft durchaus umstrittene - Methoden zur Verfügung (für eine kritische Übersicht vgl. Sumner 1996).

2. Das Maß der Präferenzbefriedigung stellvertretend für die objektive Glücksmenge anzusetzen, erscheint überdies angemessen, wenn man entweder akzeptiert, dass Glück im Kern Präferenzbefriedigung ist, oder annimmt, dass Lebewesen hinreichend zuverlässig dasjenige präferieren, was sie glücklich macht.

3. Das von Bentham vertretene rein hedonistische Glücksverständnis kollidiert mit Intuitionen, die etwa durch Nozicks Gedankenexperiment der Erlebnismaschine (experience machine) mobilisiert werden können: Hätten wir die Möglichkeit, durch neurophysiologische Stimulation von Hirnarealen langfristig intensive Glückserlebnisse zu produzieren, während unser Körper faktisch in einem Wassertank treibt, würden wir uns nach Nozicks Auffassung gegen diese Möglichkeit entscheiden. Dies zeige, dass positive Erlebnisse nicht alles sind, das wir in unserem Leben als erstrebenswert betrachten (Nozick 1974, S. $42 \mathrm{ff}$.).

4. Die Orientierung an den Präferenzen der Betroffenen vermeidet kontroverse oder bevormundende (`paternalistische`) Annahmen über die relative Wichtigkeit verschiedener Glücksgüter für das subjektive Wohlbefinden Anderer. Sie scheint damit auch eher der Bedeutung persönlicher Authentizität und Autonomie für das individuelle Glück Rechnung zu tragen als der hedonistische Glücksutilitarismus.

Probleme des Präferenzutilitarismus: Umgekehrt sieht sich jedoch auch der Präferenzutilitarismus schwerwiegenden Einwänden ausgesetzt. Einerseits scheint unplausibel, dass Präferenzbefriedigung als solche nicht nur ein Gut, sondern das einzige intrinsische Gut darstellt. Andererseits ist Präferenzbefriedigung nicht so eng an das (hedonistisch interpretierte) Glück gekoppelt wie häufig angenommen wird. Wir können uns in dem, 
was uns glücklich macht, täuschen - und wir tun dies tatsächlich ziemlich häufig (Haybron 2003). Unsere Präferenzen sind stark durch die zeitliche Entfernung möglicher Glückserfahrungen beeinflusst. Auch kann das Spektrum unserer Präferenzen und Hoffnungen unter dem Eindruck von Deprivationserfahrungen verarmen, so dass die Befriedigung unserer Präferenzen unsere Glücksmöglichkeiten nicht erschöpft. Wir können Neigungen haben, die irrelevant für unser Wohlbefinden oder diesem abträglich sind. Auch muss die Intensität unserer Verlangen nicht der Intensität des Glückserlebens entsprechen, das aus der Befriedigung dieser Verlangen entspringt (Sidgwick 1903, S. 340). Die Kontroverse zwischen Glücks- und Präferenzutilitaristen dauert gegenwärtig an. Darüber hinaus werden in der weiteren (auch nicht-utilitaristischen) Diskussion auch noch andere Ansätze vertreten; z. B. solche, die Wohlbefinden am Zugang zu bestimmten Grundgütern oder dem Vorliegen bestimmter Fähigkeiten festmachen: Crisp 2006, Kap. 4; Griffin 2000; Haybron 2013; Sen/Nussbaum 1993; Sumner 1996).

Gesamt- oder Durchschnittsnutzen: Beim klassischen Utilitarismus Benthams handelt es sich um einen Gesamtnutzenutilitarismus (GNU). Das bedeutet, dass der von der Ethik als maßgeblich ausgewiesene Nutzen sowohl dadurch erhöht werden kann, dass das Nutzenniveau bereits existierender glücksfähiger Lebewesen erhöht wird, als auch dadurch, dass die Anzahl solcher Wesen erhöht wird. Der Durchschnittsnutzenutilitarismus (DNU) fordert demgegenüber die Maximierung des durchschnittlichen Nutzenniveaus aller Betroffenen. Beide Positionen haben sowohl plausible als auch unplausible Konsequenzen, wie sich insbesondere im Hinblick auf die Ethik des Bevölkerungswachstums zeigt. Zu den unplausiblen Konsequenzen des GNU gehört, dass es die effizienteste Strategie zur Nutzenmaximierung und damit moralisch geboten sein könnte, die Zahl der nutzenrelevanten Lebewesen extrem zu erhöhen, selbst wenn das individuelle Nutzenniveau dieser in einer dichtbevölkerten Welt zusammengedrängten Lebewesen sehr gering wäre - was Parfit als "abstoßende Schlussfolgerung" (repugnant conclusion) bekannt gemacht hat (Parfit 1984, Kap. 17; Tännsjö/Ryberg 2004). Zu den extrem unplausiblen Konsequenzen des DNU gehört, dass der Durchschnittsnutzen anscheinend einfach erhöht werden kann, indem Lebewesen mit (erwartbar) unterdurchschnittlicher individueller Nutzenbilanz am Entstehen gehindert oder gar getötet werden (Henson 1971; Carson 1983). Nach Auffassung von Kritiker/innen des Utilitarismus sind beide unplausiblen Konsequenzen auf dasselbe Problem zurückzuführen: Insofern der gütermonistische Utilitarismus die Maximierung des aggregierten Nutzens oder Durchschnittsnutzens als einziges Handlungsziel postuliert, kann er auch die Existenz (oder Nichtexistenz) von nutzenfähigen Lebewesen lediglich instrumentell wertschätzen. Lebewesen wären demnach um des von ihnen erlebten Glücks oder der Befriedigung ihrer Präferenzen willen da, nicht umgekehrt. Diese Auffassung lässt sich mit den Annahmen unserer Alltagsmoral kaum in Einklang bringen.

Instrumenteller Wert der Existenz? 
Heuristische und normative Prima-facie-Regeln

\section{Akt- versus Regelutilitarismus}

Akt-Utilitarismus und 'Daumenregelnı: Der klassische Akt- bzw. Handlungsutilitarismus beurteilt die moralische Richtigkeit einer Handlung unmittelbar nach Maßgabe ihres konkret erwartbaren Beitrags zum Gesamtnutzen. Idealerweise müssten also alle Akteur/innen in jedem Moment sämtliche nutzenrelevanten Konsequenzen sämtlicher verfügbaren Handlungsoptionen kalkulieren. Dies ist jedoch offenbar nicht zu leisten (Hare 1981, Kap. 3). Schon Bentham selbst hält deshalb die Orientierung an verallgemeinernden Daumenregeln für sinnvoll. Handlungsutilitaristen wie Bentham, Smart und letztlich auch Hare verstehen solche Regeln jedoch nur als heuristische Hilfsmittel, die einer zeiteffizienten Annäherung an die richtige (nutzenoptimale) Handlungsoption dienen. Darüber hinaus haben diese Regeln jedoch kein normatives Gewicht. Das heißt, dass immer dann, wenn eine zuverlässigere Folgenkalkulation erweist, dass die Regelbefolgung im konkreten Einzelfall voraussichtlich suboptimal ist, das Festhalten an der betreffenden Regel moralisch falsch wäre. Objektiv richtig bleibt immer die Einzelhandlung, die den Gesamtnutzen maximiert. Der Handlungsutilitarismus versteht moralische $>$ Regeln also nicht nur als Prima-facie-Regeln, die (im Gegensatz zu absoluten Regeln, die keinerlei Ausnahme dulden) unter Umständen zurückgestellt werden dürfen oder gegebenenfalls auch zurückgestellt werden müssen. Er versteht sie darüber hinaus auch als Prima-facie-Regeln mit rein heuristischer Funktion, nicht als Regeln mit eigenem normativem Gewicht.

\section{Heuristische und normative Prima-facie-Regeln}

Der Unterschied sei am Lügenverbot illustriert: Wer das Lügenverbot als eine Prima-facie-Regel mit genuiner normativer Bedeutung versteht, nimmt zwar an, dass es Situation geben kann, in denen es erlaubt ist, zu lügen. Zugleich nimmt er jedoch an, dass an einer lügenhaften Handlung grundsätzliches etwas Schlechtes ist, das gewissermaßen nur aufgewogen werden, aber nie verschwinden kann. Das mag sich etwa darin äußern, dass der Belogene eine Rechtfertigung, Entschuldigung oder Kompensation fordern kann. Versteht man das Lügenverbot hingegen nur als eine heuristische Prima-facie-Regel, versteht man sie bloß als Grund für die Vermutung, dass die Handlung wahrscheinlich falsch ist (im Fall des Utilitarismus zum Beispiel deshalb, weil Lügen in der Mehrzahl der Fälle nicht zur Nutzenmaximierung beiträgt). Falls diese Vermutung jedoch in einer konkreten Situation nicht zutrifft, bleibt von der vermuteten moralischen Falschheit der Handlung nichts mehr übrig. In beiden Fällen sind Prima-facie-Regeln also Gründe, die möglicherweise durch andere Gründe aufgewogen werden können. Im ersten Fall handelt es sich jedoch um Sachgründe für die moralische Richtigkeit oder Falschheit einer Handlung selbst, im zweiten Fall lediglich um Erkenntnisgründe für die Annahme, dass eine Handlung richtig oder falsch ist. 
Regelutilitarismus: Es wird kontrovers diskutiert, ob Mill moralische Regeln nur als heuristische Daumenregeln betrachtet oder ob er ihnen eine darüber hinausgehende Rolle zugewiesen und eine regelutilitaristische Position vertreten hat (zur Diskussion West 2006). Anders als der Handlungsutilitarismus bemisst der Regelutilitarismus die moralische Richtigkeit einer konkreten Handlung nicht unmittelbar an ihrem Beitrag zur Maximierung des Gesamtnutzens. Vielmehr ist eine Handlung genau dann richtig, wenn sie einer universellen Regel folgt, die (bzw. Teil eines Systems moralischer Regeln ist, das) bei (hinreichend) allgemeiner Befolgung den Gesamtnutzen maximieren würde (Brandt 1965; Hooker 2000; zur Übersicht Hooker 2016).

\section{universell - singulär / generell - spezifisch}

Insofern Aufforderungen oder Handlungsregeln singuläre Termini wie Eigennamen (Thea Schulz), demonstrative Ausdrücke (diese Handlung hier) oder konkrete Orts- oder Zeitangaben enthalten (»Thea Schulz ist verpflichtet, die Wahrheit zu sagen", "Anwälte müssen in Wuppertal Schokoladeneis essen", »Niemand darf diesem Kaninchen etwas zuleide tun«) sind sie nicht universell, sondern singulär. Diese Unterscheidung ist eine kategoriale Unterscheidung, die keine Grade zulässt. Allerdings können Handlungsregeln jeweils in mehreren Hinsichten singulär oder universell sein (z. B. bezüglich des Regeladressaten, der Handlungssituation, der Handlungsbetroffenen etc.). Insofern universelle Termini eine mehr oder weniger große Klasse von Einzeldingen unter sich befassen können, sind Handlungsregeln, insofern sie universell sind, zugleich mehr oder weniger generell bzw. spezifisch (so ist die Regel »Anwälte dürfen nicht lügen« im Hinblick auf den Normadressaten spezifischer als die Regel »Menschen dürfen nicht lügen« und weniger spezifisch als die Regel »Gelb gekleidete Anwälte dürfen nicht lügen«).

Von Universalisierung wird in der Ethik in verschiedener Bedeutung gesprochen (vgl. Potter/Timmons 1985; Singer 1963; Wimmer 1980). In einem schwachen Sinn kann Universalisierung lediglich bedeuten, eine bestimmte singuläre praktische Aussage (»Ich darf dieses Stück Kuchen nehmen«) aus einer universell formulierten Regel herzuleiten, für die vom Sprecher Gültigkeit beansprucht wird (»Bei Geburtstagsfesten, bei denen für alle Teilnehmer/innen mehrere Kuchenstücke identischer Qualität zur Verfügung stehen, darf jede/r Teilnehmer/in sich ein Stück nehmen«). In einem anspruchsvolleren Sinn wird Universalisierung dort verstanden, wo zusätzlich die universelle Zustimmungsfähigkeit (Konsensfähigkeit) derjenigen Regel gefordert wird, aus der die singuläre Aussage herzuleiten ist (so dass die folgende Aussage wahr wäre: »Alle potentiell Betroffenen können der Regel zustimmen, dass bei Geburtstagsfeiern, bei denen für alle Teilnehmer/innen... etc.«).

Die Handlungsorientierung erfolgt hier also in einem zweistufigen Verfahren. Im ersten Schritt muss geprüft werden, welche Regeln bzw. welches Regelsystem bei (hinreichend) allgemeiner Befolgung die besten Konsequenzen hätte. Im zweiten Schritt muss die für die betreffende Handlungssituation einschlägige Regel befolgt werden. Unterschiede zwi-

Nutzen allgemeiner Regelbefolgung 
Handlungsutilitarismus vs. Regelfetischismus schen verschiedenen Konzeptionen des Regelutilitarismus können sich unter anderem im Hinblick auf die Frage ergeben, ob einzelne Handlungsregeln oder gesamte Regelsysteme geprüft werden müssen, welcher Grad der Regelbefolgung als hinreichend gilt, und ob moralische Regeln als absolute Regeln oder als normative Prima-facie-Regeln verstanden werden, die genau dann, wenn ein regelkonformes Handeln dem Gesamtnutzen in gravierender Weise abträglich wäre, zurückgestellt werden dürfen. Der Regelutilitarismus scheint eher geeignet als der Handlungsutilitarismus, allgemeine Rechte oder personale Pflichten zu begründen. Denn die Befolgung allgemeiner Regeln die vorsehen, Versprechen zu halten, wahrhaftig zu sein, niemanden an der Äußerung seiner Meinung zu hindern, oder nur im Fall von Notwehr zu töten wäre mit hoher Wahrscheinlichkeit nutzenförderlicher als die Befolgung von Regeln, die Lügen und Vertragsbruch generell erlauben. Insofern der Regelutilitarismus die Richtigkeit von Handlungen nicht unmittelbar im Hinblick auf deren konkreten Nutzen im Einzelfall bestimmt, sondern von der Konformität mit moralischen Regeln abhängig macht, scheint er auch eher der alltagsmoralischen Intuition entgegenzukommen, dass manche Handlungen san sich moralisch gut oder schlecht sind, d. h. aufgrund des in ihnen verkörperten Handlungstyps.

Handlungsutilitaristische Einwände: Aus handlungsutilitaristischer Sicht erscheint der Regelutilitarismus freilich inkonsistent: Entweder müsse er fordern, dass Regeln auch dann zu befolgen sind, wenn sie nicht nutzenmaximierend seien. Das laufe jedoch auf einen Regelfetischismus hinaus, der unplausibel sei. Denn so lange an der Annahme festgehalten werde, dass das einzige moralisch relevante Gut im durch die Regelbefolgung produzierten Nutzen liege, sei gar nicht einzusehen, warum im Einzelfall Nutzenverzichte hingenommen oder sogar geboten sein sollten. Oder der Regelutilitarismus müsse für solche Fälle eine Orientierung direkt am Nutzenprinzip oder auch an spezifischeren, die besonderen Situationsumstände berücksichtigenden und dadurch auch bei allgemeiner Befolgung nutzenförderlicheren Regeln zulassen. Dann falle der Regelutilitarismus aber letztlich auf den Handlungsutilitarismus zurück (Lyons 1965; Smart 1973). Regelutilitaristen wie Richard B. Brandt entgegnen diesem Einwand mit dem Hinweis, dass schon aufgrund der beschränkten Kapazitäten zur Folgenkalkulation und zur Internalisierung moralischer Grundsätze Handlungsregeln benötigt werden, die einfach zu behalten sind und eine rasche und eindeutige Orientierung ermöglichen. Fraglich ist allerdings, ob mit solchen pragmatischen Argumenten Regeln begründet werden können, die mehr als einen heuristischen Wert haben - Regeln, die ein eigenes normatives Gewicht haben, das im Einzelfall auch gegen Nutzenerwägungen in die Waagschale geworfen werden kann. Wäre das nicht der Fall, bestünde zwischen Regel- und einem aus pragmatischen Gründen mit (je nach kognitiven Kapazitäten mehr oder weniger komplexen; vgl. Hare 1981) Daumenregeln ausgestatteten Handlungsutilitarismus tatsächlich kein Unterschied.

Regelutilitarismus als Mischtheorie: Letztlich scheint der Regelutilitarismus eine instabile Position zu beziehen. Denn so lange er an der gütermonistischen Annahme festhält, dass Moral letztlich ausschließlich am 
Ziel der Nutzenmaximierung orientiert ist, scheint er nicht begründen zu können, warum die Befolgung moralischer Regeln als solche richtig oder wertvoll sein sollte. Auch den moralischen Regeln bzw. der Regelbefolgung könnte er dann nur einen instrumentellen Wert zuschreiben. Auch der Umstand, dass allein durch die Orientierung an Regeln soziales Vertrauen etabliert oder eine effiziente soziale Handlungskoordination ermöglicht wird, bezöge seine Kraft aus der Tatsache, dass Vertrauen und soziale Handlungskoordination wiederum der Nutzenmaximierung dienen. Auf dieser Basis ließe sich jedoch nicht begründen, dass wir uns auch dann an Regeln halten sollen, wenn wir entweder sicher sein können, dass ein in der konkreten Handlungssituation nutzenmaximierender Regelverstoß unentdeckt bliebe oder die relative Gefährdung von Vertrauen und Erwartungssicherheit durch den konkreten Nutzengewinn des Regelverstoßes überkompensiert würden. Die Annahme, dass der Regelbefolgung ein eigenes moralisches Gewicht zukommt, das sich in ihrem instrumentellen Beitrag zur Nutzenmaximierung nicht erschöpft, wäre unvereinbar mit dem strikten Gütermonismus, wie er vom klassischen Utilitarismus vertreten wird. Sie scheint sich eher aus nicht-konsequentialistischen Intuitionen zu speisen. Der Regelutilitarismus lässt sich insofern am ehesten als eine Mischtheorie verstehen, die sich bemüht, widerstreitende Intuitionen zu vereinbaren, ohne sie jedoch aus einer gemeinsamen Grundlage herleiten zu können.

Ausblick: Der Utilitarismus ist im Kern eine einfache Theorie. Dem klassischen (Handlungs-, Glücks- und Gesamtnutzen-)Utilitarismus zufolge ist eine Handlung genau dann moralisch richtig, wenn sie den größtmöglichen Beitrag zum subjektiven Wohlbefinden aller betroffenen Wesen liefert. Da die Richtigkeit aller Handlungen an einem einzigen Prinzip zu bemessen ist, können sich keine Normenkonflikte ergeben. Da das utilitaristische Moralprinzip zudem nur einen einzigen intrinsischen Wert anerkennt (subjektives Wohlbefinden), sind Entscheidungsprobleme durch inkommensurable Werte ebenfalls ausgeschlossen. Der klassische Handlungsutilitarismus besticht durch konzeptionelle Klarheit und theoretische Sparsamkeit. Wertmonismus, interindividuelle Nutzenaggregation und teleologische Struktur der Theorie sorgen zusammengenommen dafür, dass sich alle moralischen Probleme in Optimierungsaufgaben übersetzen lassen. Diese Merkmale erleichtern auch die Kooperation zwischen utilitaristischer Ethik, Spieltheorie und Ökonomie. Damit erklären sie zu einem guten Teil auch den großen und andauernden Einfluss utilitaristischen Denkens. Mit der theoretischen Sparsamkeit und Klarheit der Grundlagen des klassischen Utilitarismus geht freilich eine Reihe anwendungsbezogener Fragen einher. Einige seiner praktischen Implikationen sind mit weit verbreiteten moralischen Intuitionen kaum vereinbar oder erscheinen gar anstößig. Dies gilt vor allem für die möglichen Konsequenzen der Indifferenz des utilitaristischen Maximierungsgedankens gegenüber der interindividuellen Verteilung von Nutzen und Schaden. Fraglich erscheint auch, ob der utilitaristische Konsequentialismus den normativen Ansprüchen basaler Rechte, der persönlichen Integrität, Authentizität und Verantwortlichkeit angemessen Rechnung tragen kann. 
Die moralphilosophische Diskussion hat allerdings zu einer erheblichen Diversifizierung utilitaristischer Ethiken beigetragen, die Allgemeinaussagen über >den` Utilitarismus nur bedingt sinnvoll erscheinen lässt. Auch wenn sich der Utilitarismus heute kaum mehr als geschlossene politische Reformbewegung präsentiert, sind utilitaristische Überlegungen und Modelle weiterhin von großer Bedeutung auch über die moralphilosophische Fachdiskussion hinaus; insbesondere in der Ökonomie und in politischen Planungskontexten. Die grundsätzlichen Einwände gegen den Utilitarismus sind jedoch nie verstummt. Ein Ansatz, der explizit als Gegenentwurf zum Utilitarismus konzipiert wurde, wird im übernächsten Kapitel behandelt (siehe Kap. 7).

\section{Siglenverzeichnis}

SEP - Stanford Encyclopedia of Philosophy (Zalta)

\section{Einführende Literatur}

Birnbacher, Dieter: Utilitarismus. In: Düwell, Marcus/Hübenthal, Christoph/Werner, Micha H. (Hrsg.): Handbuch Ethik. Stuttgart/Weimar 2011, S. 95-107.

Birnbacher, Dieter: Analytische Einführung in die Ethik. Berlin/New York ${ }^{3} 2013$, insbes. Kap. 5-7.

Crimmins, James E. (Hrsg.): The Bloomsbury Encyclopedia of Utilitarianism. London/New York 22017.

Eggleston, Ben/Miller, Dale E. (Hrsg.): The Cambridge Companion to Utilitarianism. Cambridge/New York 2014.

Mill, John St.: Utilitarianism. In: Ders.: Collected Works. Toronto 1963 ff., Bd. 10, S. 203-259.

Nida-Rümelin, Julian: Kritik des Konsequentialismus. München 1993.

Scarre, Geoffrey: Utilitarianism. London/New York 1996.

Sidgwick, Henry: The Methods of Ethics. London ${ }^{7} 1907$.

Singer, Peter: Practical Ethics. Cambridge/New York ${ }^{3} 2011$.

Smart, J. J.C./Williams, Bernard: Utilitarianism: For and Against. Cambridge 1973.

\section{Zitierte und weiterführende Literatur}

Ashford, Elizabeth: »Utilitarianism, Integrity, and Partiality«. In: The Journal of Philosophy 97/8 (2000), S. 421-439.

Bentham, Jeremy: The Rationale of Reward. London 1830.

Bentham, Jeremy: An Introduction to the Principles of Morals and Legislation [1789]. Oxford 1907.

Brandt, Richard B.: »Toward a Credible Form of Utilitarianism«. In: Castañeda, Hector-Neri/Nakhnikian, George (Hrsg.): Morality and the Language of Conduct. Detroit 1965, S. 107-143.

Carson, Thomas L.: "Utilitarianism and the Wrongness of Killing«. In: Erkenntnis 20/1 (1983), S. 49-60.

Copp, David (Hrsg.): The Oxford Handbook of Ethical Theory. Oxford 2006.

Crisp, Roger: Reasons and the Good. Oxford/New York 2006.

Gert, Bernard: Morality: Its Nature and Justification. New York/Oxford 1998.

Griffin, James: Well-Being: It's Meaning, Measurement and Moral Importance. Oxford/New York 2000.

Hare, Richard M.: Moral Thinking: Its Levels, Method, and Point. Oxford/New York 1981.

Haybron, Daniel M.: The Pursuit of Unhappiness: The Elusive Psychology of Well-Being. Oxford 2008.

Haybron, Daniel M.: Happiness: A Very Short Introduction. Oxford/New York 2013. 
Henson, Richard G.: »Utilitarianism and the Wrongness of Killing«. In: The Philosophical Review 80/3 (1971), S. 320-337.

Hooker, Brad: Ideal Code, Real World: A Rule-Consequentialist Theory of Morality. Oxford/New York 2000.

Hooker, Brad: »Rule Consequentialism«. In: SEP 2016.

Knight, Frank H: Risk, Uncertainty, and Profit. Boston 1921.

Kymlicka, Will: Contemporary Political Philosophy: An Introduction. Oxford/New York 2002.

Lyons, David: Forms and Limits of Utilitarianism. New York/Oxford 1965.

Mill, John St.: Collected Works. Toronto $1963 \mathrm{ff}$.

Moore, George E.: Principia Ethica [1902]. Cambridge ${ }^{8} 1959$.

Moseley, Daniel D.: »Revisiting Williams on Integrity«. In: The Journal of Value Inquiry 48/1 (2014), S. 53-68.

Nozick, Robert: Anarchy, State, and Utopia. New York 1974.

Parfit, Derek: Reasons and Persons. Oxford 1984.

Pettit, Philip: »Analytical Philosophy«. In: Goodin, Robert E./Pettit Philip/Pogge, Thomas (Hrsg.): A Companion to Contemporary Political Philosophy. Malden MA 1993, S. 5-35.

Potter, Nelson T./Timmons, Mark: Morality and Universality: Essays on Ethical Universalizability. Dordrecht u. a. 1985.

Sayre-McCord, Geoffrey: "Mill's >Proof^ of the Principle of Utility: A More than Half-Hearted Defense«. In: Social Philosophy and Policy 18/2 (2001), S. 330360 .

Sen, Amartya/Nussbaum, Martha: The Quality of Life. Oxford/New York 1993.

Shaw, William H.: Contemporary Ethics: Taking Account of Utilitarianism. Malden/Oxford 1999.

Sidgwick, Henry: Lectures on the Ethics of T. H. Green, Mr. Herbert Spencer, and J. Martineau. London 1903.

Singer, Marcus G.: Generalization in Ethics: An Essay in the Logic of Ethics, with the Rudiments of a System of Moral Philosophy. London 1963.

Slote, Michael/Pettit Philip: „Satisficing Consequentialism«. In: Proceedings of the Aristotelian Society 58 (1984), S. 139-163.

Smart, J. J. C.: »An Outline of a System of Utilitarian Ethics«. In: Smart, J. J. C./ Williams, Bernard: Utilitarianism: For and Against. Cambridge 1973, S. 3-74.

Sumner, L. W.: Welfare, Happiness, and Ethics. Oxford/New York 1996.

Tännsjö, Torbjorn/Ryberg, Jesper (Hrsg.): The Repugnant Conclusion: Essays on Population Ethics. Dordrecht u. a. 2004, S. 7-22.

Walker, A. D. M.: »Negative Utilitarianism«. In: Mind 83/331 (1974), S. 424-428.

West, Henry R. (Hrsg.): The Blackwell Guide to Mill’s Utilitarianism. Malden/ Oxford 2006.

Williams, Bernard: »A Critique of Utilitarianism«. In: Smart, J. J. C./Williams, Bernard: Utilitarianism: For and Against. Cambridge 1973, S. 73-150.

Wimmer, Reiner: Universalisierung in der Ethik: Analyse, Kritik und Rekonstruktion ethischer Rationalitätsansprüche. Frankfurt a. M. 1980.

Zalta, Edward N. (Hrsg.): The Stanford Encyclopedia of Philosophy. In: https:// plato.stanford.edu/. 
Open Access Dieses Kapitel wird unter der Creative Commons Namensnennung 4.0 International Lizenz (http://creativecommons.org/licenses/by/4.0/deed.de) veröffentlicht, welche die Nutzung, Vervielfältigung, Bearbeitung, Verbreitung und Wiedergabe in jeglichem Medium und Format erlaubt, sofern Sie den/die ursprünglichen Autor(en) und die Quelle ordnungsgemäß nennen, einen Link zur Creative Commons Lizenz beifügen und angeben, ob Änderungen vorgenommen wurden.

Die in diesem Kapitel enthaltenen Bilder und sonstiges Drittmaterial unterliegen ebenfalls der genannten Creative Commons Lizenz, sofern sich aus der Abbildungslegende nichts anderes ergibt. Sofern das betreffende Material nicht unter der genannten Creative Commons Lizenz steht und die betreffende Handlung nicht nach gesetzlichen Vorschriften erlaubt ist, ist für die oben aufgeführten Weiterverwendungen des Materials die Einwilligung des jeweiligen Rechteinhabers einzuholen. 